\title{
Semiosis icónica en el desarrollo del lenguaje infantil
}

\author{
Miguel González Pereira \\ Universidade de Santiago de Compostela \\ Facultade de Humanidades \\ Av. Filarmónica Lucense s/n \\ 27002 Lugo \\ E-mail: miguel.gonzalez.pereira@usc.es
}

\section{SEMIOSIS ICÓNICA EN EL DESARROLLO DEL LENGUAJE INFANTIL}

Publicaciones

RESUMEN reivindican la

relevancia de lo icónico en la adquisición lingüística, pero la iconicidad suele identificarse como relación de semejanza entre expresión y contenido, remitiendo asi a una visión simplificadora de la doctrina de Peirce. El objeto de la semiótica peirceana no son los signos sino la semiosis como único proceso posible de representación cognitiva. Distingue asi tres categorias fenomenológicas básicas, a la primera de las cuales (Firstness) se vincula la semiosis icónica basada en las cualidades de lo percibido. En este trabajo proponemos una lectura de Peirce que atienda a su visión del iconismo como fundamento de toda semiosis y revisamos acercamientos recientes a la iconicidad. Esta visión de la iconicidad la proyectamos al análisis de las peculiaridades de los procesos fónicos $y$ al papel de las repeticiones en el lenguaje infantil mediante la explotación del corpus Koiné, planteando su posible interés en los estudios de lingüistica clínica.

Palabras Claves: semiótica; Peirce; iconicidad; cognición corpórea; lenguaje infantil.

SUMARIO: 1. Introducción. 2 . Semiosis icónica. 3. Iconicidad en la psicologia cognitiva. 4 Semiosis icónica en el lenguaje infantil. 5. Conclusiones.

\section{ICONIC SEMIOSIS IN THE DEVELOPMENT OF CHILD LANGUAGE}

ABSTRACT: Recent papers claim the relevance of iconicity in linguistic acquisition, but it is usually identified as a relationship of similarity between expression and content, thus referring to a simplifying view of Peirce's doctrine. The object of Peircean semiotics is not signs but semiosis as the only process of possible cognitive representation. It's from this approach that he distinguishes three basic phenomenological categories, the first of which (Firstness) is linked to the iconic semiosis based on the qualities of the perceived. In this paper we propose a reading of Peirce's semiotics that attends to his vision of iconism as the foundation of all semiosis and we review recent approaches to iconicity. We project this vision of iconicity to the analysis of the peculiarities of phonic processes and to the role of repetitions in child language through the exploitation of the Koine corpus, raising its possible interest in clinical linguistics studies.

KEY WORDS: semiotics; Peirce; iconicity; embodied cognition; child language.

SUMMARY: 1. Introduction. 2 . Iconic semiosis. 3. Iconicity in cognitive psychology. 4. Iconic semiosis in child language. 5 . Conclusions.

\section{SEMIOSIS ICONIQUE DANS LE DEVELOPPEMENT DU LANGAGE DE L'ENFANT}

RÉSUMÉ: Plusieurs articles récents affirment la pertinence de l'iconique dans l'acquisition linguistique, mais l'iconicité est généralement identifiée comme une relation de similitude entre l'expression et le contenu, se référant ainsi à une vision simplificatrice de la doctrine de Peirce. L'objet de la sémiotique de Peirce n'est pas les signes mais la sémiose comme seul processus possible de représentation cognitive. Il distingue ainsi trois catégories phénoménologiques, dont la première (Firstness) est liée à la sémiose iconique basée sur les qualités de la perception. Dans cet article, nous proposons une lecture de Peirce qui répond à sa vision de l'iconisme comme fondement de toute sémiose et nous passons en revue les approches récentes de l'iconicité. Cette vision de l'iconicité est projetée à l'analyse des particularités des processus phoniques et du rôle des répétitions dans le langage des enfants par l'exploitation du corpus Koiné, et nous suggérons son éventuelle pertinence pour la linguistique clinique.

MOTS CLÉS: sémiotique; Peirce; iconicité; cognition incarnée; langage de l'enfant.

SOMMAIRE: 1 . Introduction. 2 . Semiose iconique. 3. L'iconicité en psychologie cognitive. 4. Semiose iconique en le langage de l'enfant. 5. Conclusions
Fecha de Recepción Fecha de Revisión Fecha de Aceptación Fecha de Publicación
$05 / 02 / 2019$

$06 / 03 / 2019$

$10 / 05 / 2019$

$01 / 12 / 2020$ 


\title{
Semiosis icónica en el desarrollo del lenguaje infantil ${ }^{1}$
}

\author{
Miguel GonZÁleZ PEREIRA
}

\section{INTRODUCCIÓN}

La reivindicación de la iconicidad en el análisis del lenguaje adulto está presente desde hace décadas (cf., por ejemplo, Haiman, 1980; Givón, 1985). En estudios recientes sobre el desarrollo cognitivo se ha actualizado este énfasis en la iconicidad (cf. § 3), destacándose el papel de los elementos icónicos en los procesos de adquisición lingüística.

La iconicidad en el lenguaje adulto se aborda, justificadamente, desde la perspectiva de una iconicidad secundaria. El vínculo explicativo de la capacidad significativa de las construcciones adultas descansa en la convencionalidad establecida en el código de cada una de las lenguas y solo a partir de ese fundamento en el acuerdo social se plantea la búsqueda de aspectos que puedan mostrar motivación icónica. Desde la psicología cognitiva también se señala esta iconicidad secundaria (cf. § 3), pero solo en algunos casos se apunta el reconocimiento de una iconicidad primaria en las fases iniciales del desarrollo cognitivo y lingüístico.

En este trabajo queremos acercarnos a estos enfoques partiendo de una relectura del concepto de iconicidad en Peirce. Para ello será necesario desbrozar los muy diversos modos como se ha entendido la iconicidad peirceana, diferenciando entre el iconismo puro, estrechamente vinculado a la categoría fenomenológica de la Firstness, y los tipos de signos icónicos en los que coexistirán distintos fundamentos semióticos. En esta aproximación semiótica a la iconicidad intentaremos resaltar la necesidad de diferenciar su muy distinto papel en el lenguaje adulto y en el lenguaje infantil. Si entendemos el desarrollo lingüístico del niño como desarrollo semiótico, será preciso reconocer fases en este proceso y estas se pueden poner en relación con lo que habitualmente se ha entendido como tres tipos de signos, pero que en la obra de Peirce responde en realidad a tres modos de semiosis, a tres formas de representación cognitiva. El lenguaje verbal adulto refleja una semiosis triádica plenamente simbólica, en cuanto que sus expresiones (Representamen) significan un Objeto mediante una interpretación (Interpretant), pero lo que encontramos en el lenguaje infantil en edad temprana no es semiosis triádica simbólica sino un proceso hacia ese modo de representación cognitiva, construido sobre las bases de semiosis más sencillas como la icónica y la indéxica.

El lenguaje peculiar de los niños no debe ser entendido como reproducción deficiente del lenguaje adulto y de los signos de su código convencional sino como proceso de significación de lo que le rodea siguiendo

\footnotetext{
${ }^{1}$ Investigación desarrollada dentro de los proyectos "Procesos fónicos y emergencia de sonidos idiomáticos. Estudio de corpus" (FFI2013-41851-P) y "Adquisición fónica y corpus. Tratamiento en PHON del corpus koiné de habla infantil" (FFI2017-82752-P).
} 
unas pautas de evolución propias, que partirán de su aprehensión inicial de las propiedades de aquello que experimenta. Este primer modo de representación semiótica se corresponde con el iconismo puro y primario del que habla Peirce y sería el propio del desarrollo semiótico en edad temprana, cuando el niño está viviendo una etapa de descubrimiento e invención mediante un método hipotético (abducción).

Una vez argumentada esta consideración de la semiosis icónica como primer modo de representación cognitiva, en el siguiente apartado intentaremos seguir su rastro en algunos trabajos recientes sobre el desarrollo cognitivo. Aludiremos a la proliferación de estudios que destacan la importancia del sound symbolism y señalaremos cómo detrás de algunas propuestas de cognición corpórea se pueden encontrar ecos de la iconicidad semiótica primaria.

Terminaremos apuntando vías de aproximación a muestras de lenguaje infantil que podrian contribuir a visibilizar este desarrollo semiótico que parte de la iconicidad para caminar hacia el simbolismo codificado, concluyendo con unas breves indicaciones sobre la posible relevancia de este enfoque en los estudios de lingüística clínica.

\section{Semiosis ICónicA}

Sonesson comienza su acercamiento a una teoría general de la iconicidad con una afirmación que pone de manifiesto el espiritu de nuestro trabajo: "There is not much point using the term "iconicity" simply as a synonym for similarity. Nor is it of much use employing oneself to find out "what Peirce really thought" about iconicity, though it cannot hurt starting from here" (Sonesson, 2008: 47). Asumiendo que el carácter fragmentario de la obra de Peirce hace imposible desvelar el sentido último, y univoco, de su concepto de iconicidad, planteamos aquí una relectura que supere la simplificadora identificación con una relación de semejanza entre expresión y contenido y que sea más relevante para acercarnos al lenguaje infantil.

Peirce en ningún momento alude explícitamente a procesos semióticos desde la perspectiva del desarrollo ontogenético, por lo que no encontramos en su obra reflexiones sobre la semiosis en los niños. Para él todas las palabras del lenguaje adulto son simbolos (CP 2.292), pero en distintas ocasiones muestra que concibe la representación semiótica como un proceso en evolución y que el estadio final de los simbolos se alcanza a partir de representaciones semióticas más básicas: "Symbols grow. They come into being by development out of other signs, particularly from icons, or from mixed signs partaking of the nature of icons and symbols. We think only in signs. These mental signs are of mixed nature" (CP 2.302). De este pasaje podemos extraer algunas ideas centrales para nuestra lectura de su doctrina: 
- La representación simbólica, distintiva de la cognición humana, no surge de repente, sino que es el resultado de un desarrollo semiótico que parte de semiosis más básicas, fundamentalmente la icónica.

- Los signos son considerados en cuanto que representaciones cognitivas, como el único modo de categorizar nuestras experiencias, y no como unidades de un código comunicativo establecido.

- Estas representaciones cognitivas no son puras sino resultado de la mezcla de los tres modos de semiosis.

Este fragmento sintetiza algunos ejes básicos de la complejidad de la teoría de Peirce que han quedado ocultos bajo la simplificación de una propuesta de tres clases discretas de signos. Los elementos del lenguaje verbal adulto son símbolos, porque manifiestan la capacidad humana para una representación mediada, triádica, de sus experiencias, pero no son puramente simbólicos, porque en ellos hay rastros de semiosis icónica e indéxica. En el análisis del lenguaje adulto resulta relevante buscar rastros de esta impureza y señalar aspectos de iconicidad e indexicalidad, que tendrán en este caso carácter secundario. Pero en el lenguaje de los niños no podemos presuponer la capacidad de representación simbólica sino analizar cómo se desarrolla (Piaget, 1959) y, siguiendo la doctrina de Peirce, la iconicidad será su punto de partida.

Para abordar esto conviene partir de otra consideración crucial plasmada en este fragmento: la semiótica de Peirce es una teoría cognitiva. Cuando postula la Semiótica como disciplina de la que se siente pionero ( $C P$ 5.488), la sitúa como derivación de su teoría fenomenológica (cf. Pharies, 1985: $\S 1)$. Su ambición filosófica es construir una teoría exhaustiva, parangonable con la de Aristóteles (CP 1.1), que parta de establecer las categorias de la realidad aprehendidas por la mente humana (fenomenologia) y que explique nuestro modo de significarlas (semiótica). Su doctrina está guiada por la crítica al dualismo cartesiano y a su separación de la actividad mental de la interacción con lo que nos rodea, configurando una propuesta que avanza la defensa de una cognición corpórea frente a las teorias cognitivas formales del racionalismo y sus derivaciones. El desafio de la semiótica consiste en intentar entender cómo representamos mentalmente lo que nos rodea, porque "We have no power of thinking without signs" (CP 5.265) y "We can admit no statement concerning what passes within us except as a hypothesis necessary to explain what takes place in what we commonly call the external world" (CP 2.266). Los distintos modos de significar configuran así nuestro modo de enfrentarnos a las categorias fenomenológicas, no ontológicas, de la realidad.

Una comprensión cabal del iconismo requiere, por tanto, acercarnos a la tricotomía categorial sobre la que Peirce construye su doctrina semiótica. Asumiendo su carácter "inextricably mixed" ( $C P$ 1.286), argumenta la pertinencia de distinguir tres categorías en la descripción de nuestra aprehensión de lo que nos rodea: Firstness, Secondness, Thirdness. La 
Firstness remite a nuestro primer modo de interacción con el mundo externo y no va más allá de las sensaciones perceptivas, de las cualidades de las cosas sin relación con nada más (CP 1.531); la Secondness implica el modo de concebir algo como directamente relacionado con otra cosa, por lo que remite a lo que sucede, a las acciones ( $C P 1.24)$; la Thirdness ya supone la mediación, la interpretación generalizadora a partir de una regla establecida por hábito, por lo que entramos en el ámbito genuino de la representación cognitiva del hombre ( $C P$ 1.532). Como ya hemos visto, los signos concretos son de naturaleza mixta, pero su explicación ha de pasar por diferenciar los tres modos de significación derivados de estas tres categorias: la iconicidad que remite a la Firstness; la indexicalidad que se apoya en la Secondness y el simbolismo que constituye la manifestación genuina de la Thirdness ( $C P$ 2.299).

Dado que el iconismo puro, primario, se asienta en la Firstness vamos a detenernos un poco más en esta categoria. Como señala Sonesson (2013), en sus distintas caracterizaciones podemos encontrar una idea central que la vincula con lo presente, con la emergencia de los fenómenos, con aquello que llama nuestra atención. Estamos ante la categoría de la aprehensión sensorial en sí misma sin relación con ninguna otra cosa. Esta primera categoría fenomenológica nos restringe a un "mode of being which consists in its subject's being positively such as it is regardless of aught else. That can only be a possibility" (CP 1.25). En la interacción con el entorno la primera categoría que despierta nuestra atención cognoscitiva radica en las cualidades de lo que percibimos sin referir ni significar nada más allá de las propias sensaciones que nos provoca en ese momento (CP 1.357). Pero en esta primera categoría se activan las distintas posibilidades de relación con otras cosas, que se apoyarán inicialmente en la semejanza entre las cualidades percibidas.

El niño al nacer se sitúa en el terreno de la Firstness: este es el punto de partida del proceso cognitivo que conllevará su desarrollo semiótico. Siguiendo también en esto a Peirce, el método de conocimiento inicial no puede ser más que el hipotético, la abducción, porque es la única vía para el descubrimiento ( $C P$ 5.171). Las primeras hipótesis del niño se apoyarán necesariamente en las cualidades que percibe, en la Firstness. Su aventura abductiva, buscando la identificación de Objetos Inmediatos en el continuo de Objetos Dinámicos que le rodean, partirá de las sensaciones que provocan en su mente sus experiencias sensoriales y motoras. En el ámbito del desarrollo lingüístico, estos primeros pasos -imprescindibles para la posterior atención a la Secondness (lo que sucede) y a la Thirdness (la representación mediada por una interpretación)- nos sitúan ante las sensaciones propiciadas por las voces de sus cuidadores, por la entonación, que aún no significan nada, pero en las que sí que puede identificar las cualidades acústicas que le abrirán infinitas posibilidades de representación conforme avance su desarrollo semiótico. En este terreno de la Firstness debemos situar el iconismo puro, primario, de la teoría peirceana. 
Peirce dedica una parte importante de su obra a caracterizar los signos icónicos desvelando los fundamentos de iconicidad latentes en muchos de los simbolos de la semiosis adulta. Se trata en estos casos de una iconicidad secundaria, de lo que cataloga como hypoicons (imágenes, diagramas, metáforas), que generalmente tienen una naturaleza semiótica híbrida. Esta es la parte de su obra que ha tenido más influencia y la que está detrás de la mayoría de los estudios sobre la iconicidad en el lenguaje. Convendría, sin embargo, diferenciar entre su manera de caracterizar la iconicidad como fundamento (ground) semiótico y su análisis de los signos icónicos (Sonesson, 2008). En los signos icónicos late una iconicidad primaria que en la semiosis adulta se entremezcla con los otros modos de representación semiótica, pero que en las fases iniciales del desarrollo semiótico del niño constituiría el único mecanismo disponible para activar el proceso de significación de sus experiencias.

Esta doble perspectiva sobre el iconismo -como fundamento de una semiosis posible y como clase de signos- podemos verla reflejada en $C P$ 2.274-2.276. El signo, en cuanto que Representamen, "is a First", una cualidad en sí misma, que funcionará como signo, como representación, solo cuando establezca una relación con un Objeto mediante la determinación de un Interpretante. El iconismo puro descansa en la Firstness, en la cualidad sentida que no representa ninguna otra cosa, que no es aún Representamen de nada sino mera posibilidad. Pero esa cualidad percibida también puede ser el fundamento de una representación significativa apoyada en la semejanza. En ese momento ya estaremos ante signos, pero no serán puramente icónicos sino hypoicons, porque su semiosis triádica genuina requerirá de una interpretación establecida por convención $(C P$ 2.276).

El iconismo primario en Peirce apunta, por tanto, a una protosemiosis y a su raíz en la actividad sensorial que aún no representa nada, pero que es la posibilidad de un desarrollo semiótico. El icono puro aparece así como un Originalian sign, el grado mayor de "degeneración" sígnica, porque no representa nada y "whose significant virtue is due simply to its Quality" (CP 2.92). El icono puro "is strictly a possibility involving a possibility" ( $C P$ 2.311), que no significa ni refiere ningún objeto. El icono puro no existe como signo ( $C P$ 3.362), porque una vez que su interpretación se haya generalizado ya pasará a representar un objeto. Peirce señala que el modo de ser del iconismo puro es de carácter estrictamente mental: "It exists only as an image in the mind" (CP 4.447).

Como señalábamos, y pasajes como estos evidencian, la semiótica de Peirce es de carácter cognitivo y el fundamento de la iconicidad, más allá de su actualización en signos concretos, radica en la actividad mental. Si, como dice Sonesson (2008: 53), "iconicity is only a potential ground", el fundamento de esta posibilidad de representación semiótica está en que las cualidades que percibimos en nuestra interacción con el entorno tienen la capacidad de excitar en nuestra mente sensaciones análogas: "The Icon has 
no dynamical connection with the object it represents; it simply happens that its qualities resemble those of that object, and excite analogous sensations in the mind for which it is a likeness" (CP 2.299; cursiva mia).

La ambición filosófica de Peirce le lleva a reflexionar también sobre la dimensión fisiológica de su tricotomia categorial. En esta parte de su obra (CP 1.385-1.394) encontramos especulaciones sobre la actividad cerebral relativa a sus tres categorias y a los tres modos de representación semiótica. Respecto de los fundamentos cerebrales de la semejanza lanza la hipótesis de que "two ideas are alike so far as the same nerve-cells have been concerned" (CP 1.388). La semiótica cognitiva de Peirce no solo es corpórea por su rechazo explícito del dualismo cartesiano y por su consideración de que no cabe más conocimiento que el derivado de nuestra interacción con lo que nos rodea ( $C P$ 5.265), sino porque se lanza a aventurar asientos en la actividad cerebral que, salvadas las distancias, parecen intuir el papel de las neuronas espejo en propuestas de cognición corpórea a las que aludiremos en el siguiente apartado.

Proyectando estas reflexiones sobre el iconismo peirceano al desarrollo semiótico del niño (cf. González Pereira, 2018), debemos situarnos ante un sujeto que busca dar sentido a un sinfin de experiencias desconocidas a partir de las sensaciones que percibe en su interacción con lo que le rodea. Sus primeras hipótesis abductivas en su descubrimiento del mundo solo podrán apoyarse en las cualidades de las cosas en sí mismas (Firstness). Estas cualidades serán el soporte de una protosemiótica icónica, porque las imágenes que provocan en su mente propiciarán que pueda establecer las primeras asociaciones con aquellas otras experiencias que originan sensaciones de cualidades similares. Estas sensaciones análogas en su mente abrirán la posibilidad de los primeros procesos de representación: distintos objetos percibidos que se relacionan por su semejanza. No estamos, obviamente, ante representaciones signicas genuinas, pero este iconismo primario activará la asociación de la cualidad presente con aquellas otras que evoca por su similitud. Esa capacidad de asociar lo que percibimos con algo ausente, raíz última de la formación de un signo, se despierta en el niño con el iconismo primario. Comenzará a identificar y a categorizar sonidos por la semejanza de sus cualidades acústicas y, en la medida en que esos sonidos percibidos e identificados por su similitud se vayan asociando con las situaciones de atención conjunta en las que habitualmente ocurren, irá avanzando hacia la Secondness, hacia la semiosis indéxica. La capacidad de asociación ya no estará circunscrita a las cualidades acústicas en sí mismas, sino que se dirigirá hacia los hechos, hacia las situaciones particulares en las que suele percibir cualidades acústicas semejantes. Cuando la repetición consolide esa asociación entre propiedades acústicas semejantes y situaciones frecuentes de interacción, podrá avanzar hacia la Thirdness, hacia la semiosis simbólica. A partir de ese momento esas cualidades acústicas ya no tendrán relevancia por sí mismas, ni por aquellas otras semejantes que evocan, tampoco por su 
vínculo con acciones concretas. Solo entonces pasarán a funcionar como vehículos de representación semiótica (Representamen) que usa para exteriorizar una interpretación cognitiva compartida (Interpretant) de algún Objeto, que ya no será el Objeto Dinámico de la realidad sino el Objeto Inmediato que (re)conoce mediante un signo simbólico genuino.

Enfrentarse al desarrollo semiótico en el niño exige, por tanto, un cambio de enfoque. No se trata de analizar cómo significa la realidad poniendo el Objeto como terminus ad quem del proceso semiótico, sino colocándolo como terminus a quo. Lo presente para el niño en edad temprana es el mundo que lo rodea y las sensaciones que experimenta en su interacción con él y desde ese punto de partida tendrá que ir desarrollando su capacidad para significarlo. En González Pereira (2018) destacamos el cambio de perspectiva que subyace en Eco $(1992,1997)$ respecto de su tratado semiótico de 1975 y su relevancia para acercarnos al lenguaje infantil con una mirada nueva. De los aspectos de la teoría de Eco, que allí comentamos con más detalle, rescatamos ahora un par de observaciones. En su tipologia de modos de producción de signos (cf. Eco, 1975: § 3.6; Eco, 1992: § II) diferencia diversas actividades materiales para la producción de la expresión ("reconocimiento", "ostensión", "réplica" e "invención") y dos posibles relaciones entre tipo (type) y caso concreto (token): ratio facilis y ratio difficilis. Siguiendo esta tipología, el desarrollo semiótico del niño en las edades más tempranas solo se puede entender como un proceso de "invención", no de "réplica". No viene provisto de fábrica de ningún tipo expresivo que ya reconozca como significativo y que deba replicar mediante una ratio facilis sino que sus primeras emisiones nos sitúan en el terreno de la invención de un código regido por la ratio difficilis. El niño no puede reconocer un tipo, un modelo establecido de significación, porque el lenguaje adulto está configurado desde unas capacidades de representación semiótica triádica de las que aún no dispone. Eco (1997: § 6.15) distingue dos modalidades de semiosis que pueden ayudarnos a enfocar adecuadamente el desarrollo semiótico. En la "modalidad Alfa" el plano de la expresión se percibe por una semiosis básica que no reconoce estar ante la expresión de un signo. En la "modalidad Beta", por contra, ya se presupone que estamos ante "la expresión de una función sígnica, producida intencionalmente con la finalidad de comunicar" (ibid.: 446). Como no parece justificado creer que los niños al nacer ya saben que los estímulos acústicos que perciben son expresiones de una función sígnica establecida para comunicarles representaciones cognitivas de carácter simbólico, debemos asumir que los primeros meses de su vida giran en torno a una semiosis de modalidad Alfa. En esta fase inicial de protosemiosis, o Alfa, y en su proceso hacia una semiosis de modalidad Beta, el iconismo primario jugará un papel determinante. En sus primeras aprehensiones del input verbal su atención no se dirigirá a descubrir un significado establecido, cuando aún no podrá ni reconocer su condición sígnica, sino que se apoyará en las cualidades acústicas de lo percibido y, a partir de las relaciones de semejanza que le evoquen sus distintas experiencias 
sensoriomotrices, irá empezando a hipotetizar relaciones semióticas más complejas.

\section{ICONICIDAD EN LA PSICOLOGÍA COGNITIVA}

Las teorias dominantes durante gran parte de la segunda mitad del siglo XX caracterizaban la actividad cognitiva como un procesamiento computacional de símbolos abstractos. Desde esta perspectiva formal del cognitivismo clásico el desarrollo de nuestra capacidad representacional se llevaría a cabo de modo totalmente independiente de los sistemas sensoriales y motores. Frente a estas propuestas, a lo largo de las últimas décadas se han ido consolidando teorias cognitivas que coinciden en considerar nuestros procesos cognitivos y nuestros comportamientos como parte de la interacción dinámica de un sistema corpóreo con el entorno en el que está integrado (Gomila y Calvo, 2008: 7). Estas diversas formulaciones de lo que se conoce como cognición corpórea reivindican el papel de los sistemas sensorio-motrices en los procesos de representación, desde la convicción de que "At a basic level, embodiment extends the noncontroversial idea that we learn from experience, so semantics must be grounded in our sensations and actions" (Meteyard y Vigliocco, 2008: 294).

Aunque este nuevo marco teórico suele presentarse como una reacción contra las posturas dualistas (Richardson et al., 2008: 164) que daría continuidad a la superación del cartesianismo por parte de las distintas teorias fenomenológicas (Gomila y Calvo, 2008: 6-7; Glenberg y Gallese, 2012: 905), lo cierto es que no hemos encontrado ninguna reivindicación expresa de la figura de Peirce desde las filas de la cognición corpórea. Esto no debería sorprendernos demasiado, dada la habitual interpretación simplificadora de su obra, pero sí que resulta más sorprendente que su nombre tampoco aparezca mencionado en algunos trabajos recientes que ponen el foco en la importancia de la iconicidad para el desarrollo cognitivo y lingüístico.

Pasemos a dar cuenta ahora de algunos de los principales trabajos en esta dirección. Perniss et al. (2010) es la publicación de referencia al fijar las bases de una postura que defiende que la iconicidad es una propiedad general del lenguaje humano. La iconicidad y la arbitrariedad serian principios generales del lenguaje que responden a la adaptación de las lenguas a dos exigencias en la evolución tanto filogenética como ontogenética: la eficacia y discrecionalidad de los signos lingüísticos -la arbitrariedad- y el vínculo de las formas lingüísticas con la experiencia iconicidad- (ibid.: 11-12). El predominio del papel de la arbitrariedad en las teorías sobre adquisición lingüística respondería, así, a la hegemonía de los modelos que conciben el desarrollo conceptual y el desarrollo de las formas lingüísticas como procesos separados.

En su reivindicación del papel de la iconicidad atestiguan su frecuente presencia tanto en las lenguas orales como signadas $y$, desde una 
perspectiva más cercana a nuestros intereses, se plantean también si somos especialmente sensibles a la iconicidad, si su habitual presencia reflejaría una predisposición cognitiva a las estrategias icónicas. Con este propósito reseñan tanto los trabajos clásicos de Köhler (1929) y Sapir (1929) como réplicas experimentales más recientes que muestran asociaciones fonosimbólicas de palabras inventadas como bouba y kiki con objetos de formas redondeadas y puntiaguadas, respectivamente, en hablantes de lenguas muy diversas. Estas asociaciones de cualidades acústicas con determinados significados no serian, por tanto, específicas de algunas lenguas sino reflejo de una sensibilidad universal.

La significativa presencia de formas icónicas en las lenguas adultas es, sin duda, relevante, pero nos circunscribe a un iconismo secundario. Las posibles evidencias de una sensibilidad cognitiva hacia la iconicidad en el ser humano sí que se pueden inscribir en línea con el iconismo primario de Peirce, porque la iconicidad se plantea en estos casos como un modo de representación primario anclado en nuestra experiencia del mundo sobre la base de nuestros sistemas sensoriales y motores. Las cualidades de la percepción acústica en sí mismas serán el fundamento de nuestras primeras posibilidades de representación, iconismo primario, desde las que avanzaremos hacia sistemas de representación genuinamente simbólicos. De acuerdo con lo apuntado en Perniss et al. (2010: 12), y en consonancia con nuestra lectura de Peirce, el niño se enfrenta a percepciones y experiencias de carácter continuo, no-discreto, de naturaleza analógica y sobre esas experiencias sensoriomotrices tendrá que apoyar sus primeras hipótesis encaminadas a la representación interpretativa de entidades discretas en lo que le rodea. La predisposición cognitiva al iconismo primario se entendería así como la estrategia cognitiva inicial lógica para enfrentarnos a la representación de lo continuo, de lo analógico, a partir del reconocimiento también analógico, continuo, de semejanzas entre las cualidades de las cosas tal y como las percibimos.

En Perniss y Vigliocco (2014) se refuerzan los principios argumentales de esta defensa de la iconicidad como procedimiento cognitivo necesario que funciona como puente "from a world of experience to the experience of language". La iconicidad jugaría un papel destacado en la evolución filogenética del lenguaje al suponer un mecanismo adaptativo fundamental que propicia el desarrollo de representaciones conceptuales a partir de imitaciones, semejanzas, entre lo percibido y lo ausente, que hacen emerger el desplazamiento (ibid.: 4). Su relevancia en los procesos de adquisición lingüística también vendria dada por su importancia como herramienta para reducir la ambigüedad referencial. En el transcurso de su desarrollo semiótico el niño tiene que ir filtrando las distintas hipótesis que le surgen en la asociación de sus percepciones acústicas con los objetos y acciones que va identificando en las situaciones de atención conjunta. En ese camino, fundamentar las primeras hipótesis abductivas en la semejanza entre las cualidades de sus experiencias sensoriales y motoras seria una estrategia 
facilitadora corroborada no solo en los estudios experimentales que respaldan esa dirección sino también en la fuerte presencia de la iconicidad en el habla dirigida a los niños. La iconicidad tendria, por último, especial importancia para entender el procesamiento lingüístico desde los postulados de las teorías de la cognición corpórea con su defensa del crucial papel de las simulaciones mentales de nuestras experiencias sensoriomotrices en el desarrollo de la comprensión conceptual. Distintos estudios experimentales ponen de manifiesto que en el procesamiento lingüístico referido a experiencias sensoriales y a acciones motoras se activan en nuestro cerebro redes neuronales vinculadas con las áreas corticales del procesamiento sensoriomotriz ${ }^{2}$. Esta vinculación en la actividad cerebral entre lo que percibimos hacer y lo que hacemos a través de las neuronas espejo, y entre nuestra comprensión representacional y las correspondientes experiencias sensoriales y motoras a través de la activación de procesos neuronales similares, pone de nuevo bajo el foco el papel del iconismo primario. Si asumimos que "Meaning is derived from mental simulations / representations of perceptual and motoric experience with real-world referents" (ibid.: 8), estamos colocando el iconismo primario en la base de nuestro desarrollo semiótico.

Entre las posibles críticas a su propuesta, Perniss y Vigliocco (2014) señalan la de considerar que la iconicidad solo sería un vestigio de estadios anteriores en el desarrollo evolutivo del lenguaje, un "fossil of protolanguage" (ibid.: 9), mientras que lo distintivo del lenguaje humano sería el carácter convencional de sus simbolos. Compartiendo la réplica de las autoras, queremos señalar aquí que esa crítica solo sería aplicable al lenguaje adulto, en el que el iconismo es siempre secundario, pero que en el acercamiento al lenguaje peculiar de los niños es preciso comprender y resaltar que el iconismo no es algo secundario sino primario, porque solo orientando su atención hacia las cualidades de las cosas tal como las percibe (Firstness) podrá ponerlas en relación con cualidades percibidas semejantes ahora ausentes (desplazamiento) e hipotetizar que esas relaciones de similitud pueden servirle para identificar entidades (desambiguación referencial) en las situaciones de atención conjunta (Secondness) y avanzar hacia la representación genuinamente simbólica.

La linea argumental abierta en Perniss et al. (2010) ha tenido continuidad en trabajos que buscan delimitar el papel de la arbitrariedad en nuestro procesamiento lingüístico y tiene su formulación más explícita en Imai y Kita (2014) con su propuesta de "The sound symbolism bootstrapping hypothesis for language acquisition and language evolution". Desarrollando la fundamentación teórica de Perniss et al. (2010) y Perniss y Vigliocco (2014), describen la dificultad del proceso mediante el cual el niño tiene que identificar elementos en una experiencia continua buscando asociarlos con

\footnotetext{
2 En Meteyard y Vigliocco (2008) y en Meteyard et al. (2012) pueden encontrarse revisiones detalladas de distintas posturas sobre estas cuestiones dentro de la cognición corpórea.
} 
una forma expresiva. En este proceso cognitivo para establecer representaciones semióticas -que catalogan como inductivo, pero que responde a las características de la abducción señaladas por Peirce- tendrán que apoyarse en una serie de pistas -como son la dirección de la mirada del interlocutor o la fijación de la atención conjunta- que irán de la mano de las habilidades cognitivas disponibles en cada fase de su desarrollo. En ese proceso inferencial la habilidad biológicamente configurada para detectar el simbolismo fónico sería un factor determinante, lo que les lleva a formular los cinco postulados de su hipótesis del "sound symbolism bootstrapping" (ibid.: 4). En la argumentación de sus propuestas dan cuenta de trabajos experimentales que, aplicando distintas técnicas de observación y análisis de la actividad cerebral, mostrarían la sensibilidad al simbolismo fónico en los niños incluso en el periodo preverbal. Refieren asimismo estudios que ponen de manifiesto la mayor presencia de elementos icónicos en el habla dirigida a los niños cuando son más pequeños ${ }^{3}$. Su hipótesis formaliza, de este modo, la reivindicación de que lo que venimos llamando iconismo primario no es algo periférico y secundario en el desarrollo lingüístico en edades tempranas, sino que es el mecanismo de representación semiótica que activa y posibilita las fases posteriores. Como señalan Imai y Kita (2014: $\S 8)$, la iconicidad de las cualidades acústicas no será una estrategia útil para hipotetizar la función sígnica en todas las clases de palabras en todas las etapas del desarrollo lingüístico, pero, junto con los gestos icónicos, será el disparador que ayudará a los niños a dar el salto hacia sistemas de signos convencionales. La iconicidad se apoyaria en una capacidad biológicamente configurada en la evolución de nuestra especie para asociar el habla con informaciones procedentes de otros sentidos. Esta capacidad para la asociación intermodal sería decisiva para que en nuestros antepasados germinase la cognición semiótica, al posibilitar que se diesen cuenta de que las cualidades acústicas pueden usarse para representar algo distinto, incluyendo lo no presente.

Este vínculo de la iconicidad con la predisposición biológica para asociar informaciones de distintas modalidades sensoriales la sitúa en el ámbito de las teorias de cognición corpórea y la acerca al iconismo primario, ya que supone entenderla como primer modo de representación disponible en el desarrollo semiótico hacia la simbolización convencional. Esta perspectiva contradice planteamientos como los de Tolar et al. (2008), quienes sostienen que hasta los tres años no se evidenciaría una habilidad especialmente destacada para interpretar signos icónicos, intentando refutar el carácter

3 Laing et al. (2017) propone una explicación de la frecuencia e importancia de las onomatopeyas en el lenguaje infantil temprano que cuestionaria la relevancia de la iconicidad, poniendo el foco en los rasgos prosódicos destacados de las formas onomatopéyicas presentes en el habla dirigida a los niños. Su planteamiento como alternativa crítica al peso concedido al iconismo evidencia la identificación de iconicidad con iconismo secundario, porque los rasgos destacados en las cualidades acústicas de las palabras onomatopéyicas emitidas en el habla dirigida a los niños nos sitúan, de hecho, en el ámbito del iconismo primario: la capacidad representacional que parte de las cualidades acústicas que llaman su atención perceptiva. 
más simple del procesamiento icónico. Paradójicamente, en este trabajo sí que encontramos referencia a la autoridad de Peirce (ibid.: 225), pero sus experimentos no se dirigen hacia el iconismo primario, hacia el papel del iconismo en el propio procesamiento cognitivo y lingüístico (on line), sino a la influencia del iconismo secundario en el reconocimiento e interpretación de signos de un código establecido como es el lenguaje de signos americano (ASL).

Creemos, por tanto, que propuestas como las de Perniss et al. (2010), Perniss y Vigliocco (2014) e Imai y Kita (2014) suponen un acercamiento a la iconicidad que transciende el iconismo secundario y nos coloca ante el iconismo primario y su importante papel en el desarrollo de la capacidad semiótica en el niño. El lenguaje del ser humano adulto es de carácter simbólico, porque, salvo trastorno o déficit, manifiesta el logro cognitivo de una representación semiótica de la realidad mediada por interpretaciones establecidas por convención dentro de nuestro proceso de socialización. Lo que ya no estaría tan claro es que los procesos de representación semiótica del niño tengan desde el principio este carácter de semiosis simbólica triádica. Desde nuestra lectura de Peirce defendemos que la capacidad simbólica solo se alcanza a través de un proceso de desarrollo semiótico que parte de una protosemiosis inicial apoyada en la actividad cognitiva derivada de su interacción sensoriomotriz con el entorno, en la que serán las cualidades percibidas las que activarán, por semejanza, los primeros procesos de representación. Tomasello (2003) defiende, oportunamente, que la capacidad simbólica proporciona al ser humano un modo de representación cognitiva socially learned (Tomasello, 2003: 12) distintivo de nuestra especie y plantea el desarrollo del lenguaje como simbólico desde el principio: la adquisición del lenguaje no comenzará hasta cerca del primer año de vida cuando habilidades sociales y comunicativas como la jointattention y la intention-reading propicien el uso de los símbolos lingüísticos como representaciones sociales ${ }^{4}$. Sin cuestionar el trascendente papel de estas habilidades socio-comunicativas, creemos que el desarrollo lingüístico, entendido como desarrollo semiótico, comienza antes, aun cuando en las fases iniciales no haya lugar para representaciones simbólicas de fundamento socio-cultural, porque el niño solo puede aprehender cognitivamente las cosas que percibe en sí mismas, Firstness, y sobre este fundamento icónico establecerá sus primeras representaciones. Esta iconicidad basada en la actividad sensorio-motriz no será, sin embargo, algo ajeno al desarrollo lingüístico sino fundamento de su peculiaridad.

\footnotetext{
4 "Linguistic symbols provide human beings with a species-unique format for cognitive representation. That is, when a child learns the conventional use of linguistic symbols, what she is learning are the ways her forebears in the culture found it useful to share and manipulate the attention of others in the past. [...]. But near the end of the first year of life something new happens in the way human infants relate to other persons, and, in the current account, this explains why the acquisition of language begins when it does" (Tomasello, 2003: 20-21).
} 
Esta consideración del desarrollo semiótico del niño como proceso que parte del iconismo primario se ve plenamente reconocida en propuestas enmarcadas en la cognición corpórea como las de Gallese y Lakoff (2005) y Glenberg y Gallese (2012). En Gallese y Lakoff (2005) se defiende el fundamento corpóreo de nuestro conocimiento conceptual y su vinculación con los sistemas sensorio-motrices, caracterizando las imágenes mentales como una simulación de las acciones y percepciones que activa muchas de las neuronas comprometidas en la acción y en la percepción. Resaltan así la trascendencia del descubrimiento de los sistemas y mecanismos de las neuronas espejo -activas tanto al percibir como al ejecutar una acción-y su carácter multimodal. Desarrollando ese enfoque, en Glenberg y Gallese (2012) se propone una teoría sobre la adquisición lingüística action-based. Apoyándose en evidencias, psicológicas y neurofisiológicas, de la estrecha conexión entre los sistemas de procesamiento motor y lingüístico, reivindican la existencia y la importancia de speech mirror neurons que actuarian tanto en la percepción como en la producción del habla (ibid.: 908). En las fases iniciales del desarrollo lingüístico, la propia percepción de las cualidades acústicas será, por tanto, la que motivará el procesamiento cerebral de las primeras producciones.

\section{SEMIOSIS ICÓNICA EN EL LENGUAJE INFANTIL}

El reconocimiento de la iconicidad como peculiaridad del lenguaje infantil está presente en trabajos clásicos como Jakobson (1941) (cf. Fernández Pérez, 2014) o Slobin (1985), pero una referencia explícita al iconismo primario, en el sentido de Peirce, como primera fase en el desarrollo semiótico del niño solo la hemos encontrado en la interesante obra de van Lier (2004) (cf. González Pereira, 2018). A continuación, vamos a apuntar, brevemente, alguna de las posibles vías para rastrear la incidencia de esa fase inicial en el desarrollo semiótico que venimos llamando iconismo primario en muestras de habla infantil espontánea recogidas en el corpus Koiné.

La aproximación más obvia remite a la propia codificación de las onomatopeyas mediante “@o” en el sistema usado en CHILDES, pero queremos sugerir otras líneas de exploración. Analizar el desarrollo del componente fónico en el niño conlleva reconocer las singularidades de sus producciones. En Fernández López y Prego (2014) se señalan algunas de estas singularidades a partir de la explotación de los datos del corpus Koiné. La fase que se cataloga como "inicial", antes de los tres años, tendría entre sus rasgos peculiares la mayor estabilidad de los elementos vocálicos y la mayor conservación de sílabas tónicas y postónicas. Los datos de un corpus extenso como Koiné corroboran de este modo observaciones habituales en la caracterización de las producciones fónicas en las edades más tempranas. Lo que querriamos plantear aquí es en qué medida singularidades como estas pueden explicarse como manifestación del iconismo primario. La 
mayor conservación de las sílabas tónicas y postónicas, por ejemplo, parece responder a esta pauta.

(1) Elf1_04

ART: $1 ; 11.12$

*ART: mira máforo [//] máforo

(2) Elf1_04

RUT: 2;0.15; MON: adulto

*MON: esa es una hada madrina

*RUT: un nina

(3) Elf1_04

RUT: $2 ; 0.15 ;$ MON: adulto

*MON: ++ esto es un muñeco de madera

${ }^{*}$ RUT: nena

Para el niño de corta edad la sílaba tónica no es sino un elemento perceptivo destacado en las emisiones que recibe. La mayor intensidad actúa como una cualidad acústica que percibe e identifica con más facilidad, por lo que le resultará más fácil de producir -percepción y ejecución como actividades neurocognitivamente vinculadas en este iconismo primario-. La sílaba tónica será para él el elemento acústico percibido al que agarrarse en sus producciones guiadas por procesos fónicos específicos como los de asimilación y reduplicación consonántica ilustrados en (2) y (3). Si no evaluamos las realizaciones fónicas del lenguaje infantil temprano como reproducciones deficientes de las formas del lenguaje adulto, será más fácil reconocer estrategias específicas, adaptadas a su nivel de desarrollo semiótico, en su forma de construir fónicamente la expresión de sus signos. En ese sentido, en las primeras fases los factores que responden a la motivación icónica prevalecerán muchas veces sobre aquellos motivados por el acuerdo con la forma simbólica adulta.

(4) Elf1_01

RIC: $1 ; 10.18 ;$ MON: adulto

*MON: a ver (.) vamos a empezar desde el principio (.) esto es un $+/ /$.

*RIC: ++ guaguas [*]

*MON: un paraguas (.) y para qué se usa el paraguas? [...]

${ }^{*} \mathrm{RIC}$ : $\quad \mathrm{xxx}$ guaguas [*].

RIC no parece guiar la elaboración de su expresión fónica por la reproducción de la expresión del símbolo convencional adulto, ni siquiera cuando se le presenta como modelo, sino por mecanismos propios de su modo de significar. La forma "guaguas" no es una onomatopeya en el sentido 
tradicional, pero es claramente una producción que sigue el patrón de la iconicidad. La complejidad silábica del simbolo adulto se adapta como reduplicación de una estructura silábica ya estable en esta fase, que no solo se parece a la onomatopeya para designar al perro, sino que se asemeja a su forma de significar el agua, con la que asocia icónicamente el significado de esta forma léxica.

Muestras como las de (2), (3) y (4) nos sitúan ante otro aspecto caracterizador del habla infantil temprana que también debería enfocarse desde la perspectiva de un desarrollo semiótico enraizado en el iconismo. Nos referimos al modo de acercarnos a la proliferación de repeticiones. Estas suelen analizarse y clasificarse como repeticiones totales o parciales de la forma adulta. Esto supone partir de la consideración de que desde el principio el niño reconoce unidades de un código semiótico, de carácter simbólico, que busca replicar. En línea con lo que señalábamos al hablar de Eco (cf. González Pereira, 2018), las repeticiones en edad temprana no deberian abordarse como réplicas de símbolos establecidos, guiadas por la ratio facilis, sino como invenciones semióticas encaminadas a crear el propio type a seguir (ratio difficilis). En ese sentido hetero-repeticiones como las de estos ejemplos nos mostrarían cómo en ese proceso de "invención" prevalecería la semiosis icónica primaria. Los niños no estarian tanto reproduciendo, parcial e infructuosamente, las producciones simbólicas que se le dirigen como utilizando las cualidades acústicas que perciben, y que ya identifican por su semejanza con otras percepciones, para activar sus propias producciones. Las repeticiones de elementos acústicos, que no de signos simbólicos, responderian así a la acción de las speech mirror neurons de las que hablan Glenberg y Gallese (2012), porque, como dice van Lier (2004: 71): "Language use is primarily voice, rather than speech".

Otorgar un papel destacado a la semiosis icónica en el lenguaje infantil no solo nos ofrece un enfoque que resalta su importancia como pista que facilita el desarrollo de la capacidad representacional al asociar la expresión con la experiencia perceptiva, sino que también puede ser de gran relevancia en el ámbito de la lingüística clínica. Junto a los muchos trabajos experimentales que en los últimos años han señalado las ventajas de las formas icónicas en los procesos de adquisición lingüística, también encontramos estudios que abordan la incidencia de la iconicidad en distintos tipos de trastornos que afectan al lenguaje. Ozturk et al. (2013) aportan resultados de pruebas experimentales, con tareas de análisis de duración de miradas, que evidenciarian que los niños de cuatro meses ya serían sensibles a la asociación de ciertos sonidos con determinadas formas de los objetos, lo que reforzaría la hipótesis de la tendencia a establecer correspondencias de semejanza entre percepciones de distintas modalidades desde edades muy tempranas. Al explicar esta tendencia desde las teorias que afirman el estrecho vínculo entre los mecanismos de percepción y de producción, remiten a trabajos que muestran que no estaría igualmente presente en individuos con desórdenes del espectro autista o con 
daño cerebral, que parecen presentar disfunciones en los sistemas de neuronas espejo. En Meteyard et al. (2015), por otro lado, se estudia si las ventajas que ofrece la iconicidad en la adquisición y procesamiento lingüístico en las personas sin déficit también se da en pacientes afásicos. De su estudio concluyen que las asociaciones icónicas no solo son beneficiosas en los procesos de adquisición léxica, porque establecen un vínculo más fuerte entre la forma y el significado, sino que "iconic forms are also more robust to neurological damage. As such, there may be potential for using iconic word-forms to support language rehabilitation in aphasia" (ibid.: 274).

\section{Conclusiones}

En la descomunal obra de Peirce se entreveran las consideraciones acerca del iconismo presente en los signos simbólicos, iconismo secundario, con las reflexiones sobre el fundamento del iconismo puro en las cualidades percibidas de las cosas y su condición de origen del desarrollo semiótico posterior que conducirá hasta la representación simbólica. Este iconismo primario, que solo existe como imagen en la mente, activa el desarrollo de la capacidad representacional al excitar sensaciones análogas en virtud de la semejanza. Detrás de las actuales reivindicaciones de la iconicidad como propiedad general del lenguaje y de la defensa de su destacado papel en el desarrollo lingüístico creemos que pueden rastrearse las huellas del iconismo primario de Peirce, aun cuando no se expliciten.

La hipótesis de que la predisposición a la iconicidad sería un factor crucial para activar el desarrollo lingüístico entronca perfectamente con la visión de nuestra capacidad de semiosis simbólica como proceso que parte del iconismo. Desde las dos perspectivas se está resaltando que la actividad cognitiva tiene sus raíces en una interacción con el entorno en la que solo podemos partir de las sensaciones percibidas como base para nuestras primeras hipótesis interpretativas apoyadas en la semejanza entre percepciones provenientes de las distintas modalidades sensoriales. La actividad de los sistemas de neuronas espejo se presenta así como sustrato cerebral de un proceso cognitivo que arrancaría de la simulación mental de nuestras experiencias sensorio-motrices y daría fundamento neurológico a la intuición peirceana. En la medida en que se consoliden evidencias de la importancia de estos mecanismos icónicos en el arranque de nuestro desarrollo cognitivo y lingüístico, la lingüística clínica habrá de tenerlos en cuenta para diagnosticar los fundamentos de los distintos déficits lingüísticos y para diseñar las estrategias reparadoras más eficaces.

En el análisis del lenguaje infantil las alusiones a la iconicidad suelen destacar la gran presencia de onomatopeyas, pero estas están más cerca del iconismo secundario que del primario. El papel de este iconismo primario en el desarrollo semiótico del niño debería buscarse en las posibles muestras de rasgos de sus producciones fónicas peculiares que parezcan obedecer a 
que aún no están comprometidos con la reproducción de los símbolos del lenguaje adulto sino con la invención de su propio sistema de representación, apoyado inicialmente en el reconocimiento de las cualidades acústicas percibidas, y en su repetición.

\section{REFERENCIAS}

CP: PEIRCE, C. S. (1931-1935):

The Collected Papers of Charles Sanders Peirce. Vols. I-VI, Hartshorne, Ch. \& Weiss, P. (eds.), Cambridge, MA: Harvard University Press. Vols. VIIVIII, Burks, A. W. (1958) (ed.), Cambridge, MA: Harvard University Press.

ECO, U. (1975): Trattato di semiotica generale, Milano: Bompiani. Trad. cast. de C. Manzano, Tratado de semiótica general, Barcelona: Lumen, 1977.

ECO, U. (1992): La production des signes, Paris: Librairie Générale Française.

ECO, U. (1997): Kant e l'ornitorrinco, Milano: R.C.S. Libri. Trad. cast. de $\mathrm{H}$. Lozano, Kant y el ornitorrinco, Barcelona: Lumen, 1999.

FERNÁNDEZ LÓPEZ, I. y PREGO, G. (2014): "Exploración lingüística del habla infantil", Fernández Pérez, M. (coord.): Lingüistica $y$ déficit comunicativos, Madrid: Sintesis, pp. 45-99.

FERNÁNDEZ PÉREZ, M. (2014): "Roman Jakobson y su contribución al estudio del lenguaje peculiar", Calero, $\mathrm{M}^{\mathrm{a}}$ L. et al. (eds.): Métodos y resultados actuales en Historiografía de la
Lingüistica, Münster: Nodus Publikationen, pp. 193-203. GALLESE， V. \& LAKOFF， G. (2005): “The brain's concepts: The role of the sensory-motor system in conceptual knowledge", Cognitive Neuropsychology, 22 (3/4), pp. 455-479.

GIVÓN, T. (1985): "Iconicity, isomorphism, and nonarbitrary coding in syntax", Haiman, J. (ed.): Iconicity in Syntax, Amsterdam: John Benjamins, pp. 187-220.

GLENBERG, A. M. \& GALLESE, V. (2012): "Action-based language: A theory of language acquisition, comprehension and production", Cortex, 48 (7), pp. 905-922.

GOMILA, T. \& CALVO, P. (2008): "Directions for an Embodied Cognitive Science: Toward an Integrated Approach", Gomila, T. \& Calvo, P. (eds.): Handbook of Cognitive Science. An embodied approach, Oxford/Amsterdam: Elsevier Science, pp. 1-25.

GONZÁLEZ PEREIRA, M. (2018): "Aproximación semiótica al desarrollo del lenguaje infantil en edad temprana", Díaz, M. et al. (eds.), Actas do XIII Congreso Internacional de Lingüistica 
Xeral, Vigo: Universidade de Vigo, pp. 439-446.

HAIMAN, J. (1980): “The Iconicity of Grammar: Isomorphism and Motivation", Language, 56 (3), pp. 515-540.

IMAI, M. \& KITA S. (2014): “The sound symbolism bootstrapping hypothesis for language acquisition and language evolution", Philosophical Transactions of the Royal Society $B$ 369: 20130298.

JAKOBSON, R. (1941): Kindersprache, Aphasie und Allgemeine Lautgesetze, Uppsala: Universitets Arsskrift. Trad. cast. de E. Benítez (sobre versión francesa), Lenguaje infantil y afasia, Madrid: Ayuso.

KÖHLER, W. (1929): Gestalt Psychology. New York: Liveright.

LAING, C. et al. (2017): "How salient are onomatopoeia in the early input? A prosodic analysis of infant-directed speech", Journal of Child Language, 44, pp. 11171139.

METEYARD, L. \& VIGLIOCCO, G. (2008): “The Role of Sensory and Motor Information in Semantic Representation: A Review", Gomila, T. \& Calvo, P. (eds.): Handbook of Cognitive Science. An embodied approach, Oxford/Amsterdam:

Elsevier Science, pp. 293312

METEYARD et al. (2012): “Coming of age: A review of embodiment and the neuroscience of semantics", Cortex, 48, pp. 788-804.

METEYARD et al. (2015): "When semantics aids phonology: A processing advantage for iconic word forms in aphasia", Neuropsychologia, 76, pp. 264-275.

OZTURK et al. (2013): "Sound symbolism in infancy: Evidence for sound-shape cross-modal correspondences in 4months-olds", Journal of Experimental Child Psychology, 114, pp. 173186.

PERNISS, P. et al. (2010): "Iconicity as a general property of language: evidence from spoken and signed languages", Frontiers in Psychology 1, pp. 1-15.

PERNISS P. \& VIGLIOCCO G. (2014): “The bridge of iconicity: from a world of experience to the experience of language", Philosophical Transactions of the Royal Society B 369, 20140179.

PHARIES, D. (1985): Charles S. Peirce and the linguistic sign, Amsterdam: John Benjamins.

PIAGET, J. (1959): La formation $d u$ symbole chez l'enfant. Neuchatel: Delachaux \& Niestlé.

RICHARDSON, M. J. et al. (2008): "Ecological Psychology: Six Principles for an EmbodiedEmbedded Approach to Behavior", Gomila, T. \& Calvo, P. (eds.): Handbook of Cognitive Science. An embodied approach, 
Oxford/Amsterdam:

Elsevier Science, pp. 161187.

SAPIR, E. (1929): “A study in phonetic symbolism", Journal of Experimental Psychology 12, pp. 225-239.

SLOBIN, D. (1985): "The child as a linguistic icon-maker", Haiman, J. (ed.): Iconicity in Syntax, Amsterdam: John Benjamins, pp. 221-248.

SONESSON, G. (2008): "Prolegomena to a general theory of iconicity considerations on language, gesture, and pictures", Klaas Willems, K. \& De Cuypere, L. (eds.): $\mathrm{Na}$ turalness and Iconicity in Language, Amsterdam: John Benjamins, pp. 47-72.
SONESSON, G. (2013). "The natural history of branching: approaches to the phenomenology of firstness, secondness, and thirdness", Signs and Society, 1 (2), pp. 297-326.

TOLAR, T. et al. (2008): "The development of the ability to recognize the meaning of iconic signs", Journal of Deaf Studies and Deaf Education, 13, pp. 225-240.

TOMASELLO, M. (2003): Constructing a language, Cambridge (MA): Harvard Univ. Press.

VAN LIER, L. (2004): The Ecology and Semiotics of Language Learning. A Sociocultural Perspective, Norwell: Kluwer Academic Publishers. 